\title{
Do children and adolescents with completely resected alveolar rhabdomyosarcoma require adjuvant radiation? A report from the Children's Oncology Group
}

\author{
Jamie M. Aye ${ }^{1}$, Yueh-yun $\mathrm{Chi}^{2}$, Jing Tian ${ }^{2}$, Erin R. Rudzinski ${ }^{3}$, Odion T. Binitie ${ }^{4}$, Roshni \\ Dasgupta $^{5}$, Suzanne L. Wolden ${ }^{6}$, Douglas S. Hawkins ${ }^{7}$, Abha A. Gupta ${ }^{8}$ \\ ${ }^{1}$ Department of Pediatrics, Children's of Alabama, University of Alabama at Birmingham, \\ Birmingham, Alabama \\ ${ }^{2}$ Department of Biostatistics, College of Public Health and Health Professions College of \\ Medicine, University of Florida, Gainesville, Florida \\ ${ }^{3}$ Department of Pathology, Seattle Children's Hospital, University of Washington, Seattle, \\ Washington \\ ${ }^{4}$ Department of Surgery, Johns Hopkins All Children's Hospital, St. Petersburg, Florida \\ ${ }^{5}$ Department of Pediatric General and Thoracic Surgery, Cincinnati Children's Medical Center, \\ University of Cincinnati, Cincinnati, Ohio \\ ${ }^{6}$ Department of Radiation Oncology, Memorial Sloan Kettering Cancer Center, New York, New \\ York \\ ${ }^{7}$ Department of Pediatrics, Seattle Children's Hospital, University of Washington, Seattle, \\ Washington \\ ${ }^{8}$ Department of Pediatrics, Hospital for Sick Children, University of Toronto, Toronto, Canada
}

\section{Abstract}

Background-The role of adjuvant radiotherapy (RT) remains unclear in patients with localized. completely resected (group I) alveolar rhabdomyosarcoma (ARMS).

Procedure-: Patients with group I ARMS enrolled on any one of three prior Children's Oncology Group (COG) clinical trials (D9602, D9803, or ARST0531) were analyzed. All patients received systemic chemotherapy and 36 Gy adjuvant RT (if given) to the primary site at week 12 or week 4 for D9602/D9803 and ARST0531, respectively.

Results-Thirty-six patients with group I ARMS were treated on D9602 $(n=6)$, D9803 $(n=17)$, or ARST0531 $(n=13)$. of whom $24(67 \%)$ were male. The median age was 4.1 years (range, $0.8-$

\footnotetext{
Correspondence: Jamie M. Aye, Department of Pediatrics, Children's of Alabama, University of Alabama at Birmingham, 1600 17th Avenue South, Lowder Building, Suite 512, Birmingham, AL 35233. jaye@ peds.uab.edu. CONFLICTS OF INTEREST

The authors declare no conflicts of interest.

SUPPORTING INFORMATION

Additional supporting information may be found online in the Supporting Information section at the end of the article.
} 
45.8). Twenty (56\%) patients had an unfavorable primary site, and $10(28 \%)$ had tumors $>5 \mathrm{~cm}$. FOXOl-fusion status was negative, positive, and unknown in 10 (28\%), 15 (42\%), and 11 (30\%) tumors, respectively. Twenty-two $(61 \%)$ patients received RT. Overall, the four-year event-free survival (EFS) and overall survival (OS) were $70.8 \%$ and $88.3 \%$, respectively. Patients with FOXOI positivity who received RT had superior EFS compared with those who did not $77.8 \%$ vs $16.7 \% ; P=0.03)$. Among 10 patients who were $F O X O I$ negative, the outcome was similar with or without RT.

Conclusions-Although limited by a small sample size, data from this study support the routine use of adjuvant RT in patients with FOXOl-positive disease even after complete resection. Additionally, omitting adjuvant RT is rational for patients with FOXOl-negative ARMS and will be prospectively investigated in the current COG trial ARST1431.

\section{Keywords}

FOX01; radiation; rhabdomyosarcoma

\section{1| INTRODUCTION}

Rhabdomyosarcoma (RMS) is the most common pediatric soft-tissue sarcoma with an incidence of 0.4 cases per 100,000 children per year in the United States. ${ }^{1,2}$ Since the formation of the Intergroup Rhabdomyosarcoma Study (IRS) Group in 1972, efforts have been made to tailor therapy for patients based on prognostic factors. ${ }^{3}$ Through IRS clinical trials I-III, a surgical-pathologic grouping system was developed and associated with patient outcomes. The five-year survival rates were $93 \%, 81 \%, 73 \%$, and $30 \%$ for Groups I-IV, respectively. ${ }^{4-6}$ Furthermore, results from IRS I-III led to the development of a pretreatment staging system that accounts for primary tumor site (favorable or unfavorable), size $(<5 \mathrm{~cm}$ or $\geq 5 \mathrm{~cm}$ ), nodal involvement, and metastatic involvement. ${ }^{7}$ Favorable sites include orbit, head and neck (excluding parameningeal), genitourinary-non-bladder/prostate, and biliary tract/liver.

In addition to group and stage, patients are also risk-stratified based on tumor histology. Since IRS I, it has been established that patients with alveolar RMS (ARMS) have a poorer prognosis compared with those with embryonal RMS (ERMS). ${ }^{1,2,8}$ More recently, this relationship has been further delineated by whether the tumor is also positive for an oncogenic FOXOI fusion. Patients with FOXOl-negative ARMS have a similar outcome to patients with ERMS and superior event-free survival (EFS) compared with patients with FOXOl-positive ARMS. ${ }^{9,10}$ The latest Children's Oncology Group (COG) clinical trial, ARST1431, now incorporates $F O X O$-fusion status into a prospective risk stratification for patients with intermediate-risk RMS (NCT02567435).

The treatment for RMS is multimodal comprising of chemotherapy and surgery and/or radiotherapy (RT). Efforts have been made to reduce therapy-associated toxicities while still maintaining disease outcome. ${ }^{3} \mathrm{RT}$ is associated with a five-fold higher risk of having two or more chronic health conditions in cancer survivors. ${ }^{11}$ In patients with ERMS, dose reductions in RT did not compromise outcomes for those with microscopic residual disease. 12 In the randomized evaluation of RT on IRS I. no benefit of RT was seen in group I 
patients; however, outcome was not analyzed by histologic subtype. ${ }^{4}$ A subsequent analysis of IRS I-III demonstrated no significant benefit of RT in those with group I ERMS, and RT has since been removed from the standard treatment of these patients. ${ }^{13}$

In contrast to ERMS, the prescription of RT in ARMS, particularly in patients with completely resected group I disease, remains inconsistent. Patients with group I or II ARMS treated with intensive chemotherapy with vincristine, dactinomycin, and cyclophosphamide (VAC) and RT to $36 \mathrm{~Gy}$ had an excellent local control rate of $90 \%$ on COG D9803. ${ }^{14}$ Similarly, when patients with ARMS were treated with complex, multiagent chemotherapy (IRS III/IV), RT was of uncertain benefit in patients with group I, stage $1 / 2$ disease. ${ }^{15}$ However, RT appeared to have benefit among patients with stage 3 disease (unfavorable site, $>5 \mathrm{~cm}$, and/or regional nodal involvement). RT was recommended on subsequent COG intermediate-risk studies due to the concern for the aggressive biology of FOXOl-positive ARMS. ${ }^{16-18}$ Thus, utilizing a retrospective analysis of COG clinical trials D9602, D9803, and ARST0531, we sought to define a subset of patients with group I ARMS for whom local RT could be safely omitted without compromising outcome.

\section{2| METHODS}

\section{1 | Treatment of patients with group I ARMS on D9602, D9803, and ARST0531}

From 1997 to 1999, patients with clinical low-risk features and ARMS were eligible for enrollment on D9602, ${ }^{16}$ These patients received intensive VAC and no RT. From 1999 onward, patients with clinical low-risk features with ARMS were no longer eligible for D9602 and instead were enrolled on D9803. ${ }^{17}$ D9803 randomized patients between intensive VAC therapy or VAC alternating with cycles of vincristine, topotecan, and cyclophosphamide. Both treatment arms included 36 Gy RT for patients with group I ARMS at week 12 .

Patients enrolled on ARST0531 were randomized to receive VAC therapy or VAC with cycles of vincristine and irinotecan. ${ }^{18}$ The dose of cyclophosphamide was reduced from 2.2 $\mathrm{g} / \mathrm{m}^{2} /$ course on D9803 to $1.2 \mathrm{~g} / \mathrm{m}^{2} /$ course on ARST0531. Patients on ARST0531 were to receive 36 Gy at week 4 of therapy; however, for patients less than two years of age, individualized local control, including omission of RT, was permitted at the discretion of the treating institution.

\subsection{Patients and tumors}

All patients with group I ARMS, defined by imaging and pathology, treated on either D9602, D9803, or ARST0531 were included for analysis. Clinical variables including gender, age, stage, FOXOl-fusion status, and whether patients received RT were analyzed. Because age greater than 10 years is associated with poorer outcome for patients with localized RMS ${ }^{19}$ and because radiation could be omitted for patients less than two years of age on ARST0531, ${ }^{18}$ we subdivided age into the following categories for analysis: less than 2 , greater than 2 but less than 10, and greater than 10 years of age. All diagnoses of ARMS were established by central pathology re-review of cases using the contemporary definition of ARMS. ${ }^{20}$ 


\subsection{Statistical analysis}

Time to local failure (LF), defined as progression or relapse at the primary site as a first event (with or without concurrent regional and/or distant failure), was calculated from the time of study enrollment. Regional failure was defined as recurrence in tissue adjacent to the primary site or regional lymph nodes and distant failure was defined as the appearance of distant metastases. A competing-risks analysis was used to assess the cumulative incidence of LF, treating regional and or distant failures as competing events. ${ }^{21} \mathrm{EFS}$ was defined as the time from study enrollment to disease recurrence, second malignancy, or death, whichever occurred first. Overall survival (OS) was defined as the time from enrollment to death from any cause. The KaplanMeir method ${ }^{22}$ was used to estimate survival distributions, which were compared with the log-rank test. ${ }^{23}$ The Fisher Exact test was used to perform comparisons of categorical characteristics. ${ }^{24}$ Patient follow-up was current through December 31,2018.

\section{3 | RESULTS}

\subsection{Clinical characteristics}

Detailed clinical characteristics are listed in Table 1. Thirty-six patients with group I ARMS were treated on D9602 $(n=6)$, D9803 $(n=17)$, or ARST0531 $(n=13)$. Twenty-two (61\%) patients received RT. Twenty-four $(67 \%)$ patients were male. The median age at diagnosis was 4.1 years (range, $0.8-45.8$ ). Twenty tumors (56\%) occurred in an unfavorable site. Ten tumors $(28 \%)$ were $>5 \mathrm{~cm}$ in size, of which, four were $\mathrm{FOXOl}$ positive, two were FOXOI negative, and four had an unknown FOXOl-fusion status. Sixteen (44\%) patients had stage 1, 14 (39\%) had stage 2, and 6 (17\%) had stage 3 disease. Only one patient (from D9803) had regional node involvement $(\mathrm{Nl})$; this was seen by imaging but not by pathologic evaluation. FOXOl-fusion status was negative, positive, and unknown in 10 (28\%), 15 (42\%), and 11 (30\%) tumors, respectively.The use of RT was similar by tumor size and FOXOl-fusion status (Table 2); additionally, there were similar numbers of patients $\leq 2$ years of age who received $(n=5)$ and did not receive RT $(n=4)$.

\subsection{LF, EFS, and OS}

Four-year LF for all patients was $17.1 \%$ (95\% confidence interval [Cl]: 8.3\%-35.5\%). There was a near-significant difference $(P=0.053)$ in LF between those who received RT (fouryear LF 4.9\%; 95\% Cl: $0.9 \% 28.3 \%$ ) and those who did not (four-year LF $34.0 \%$; $95 \% \mathrm{Cl}$ : 17.4\%66.4\%). Patients who were FOXOI positive and did not receive RT had a greater number of failures than those who did receive RT (Supporting Information Table SI). Fouryear EFS and OS were 70.8\% (95\% Cl: 55.5\%-86.1\%) and 88.3\% (95\% Cl: 77.5\%-99.1\%), respectively. Ten patients experienced an event: five local-only relapses, one local + regional relapse, one regional + distant relapse, two distant relapses, and one secondary malignancy.

There was no significant difference in EFS or OS based on tumor size, stage, or FOXOIfusion status (Supporting Information Table S2). Additionally, there was no significant difference in EFS or OS based on whether patients received RT as a whole (Figure 1A and B). When stratified by $F O X O$-fusion status, the EFS was significantly improved for patients who were FOXOI positive who received RT $(n=9)$ compared with those who did not $(n=6)$ 
(four-year EFS $77.8 \%$ [95\% CI: 50.6\%-100.0\%] vs $16.7 \%$ [95\% Cl: $0.0 \%-46.7 \%$ ]; $P=$ 0.03) (Figure 2A), with a trend toward improved OS (four-year OS $100.0 \%$ vs $50.0 \%$ [95\% Cl: $10.0 \%-90.0 \%) ; P=0.08)$. In comparison, EFS and OS for patients with a negative FOXOl-fusion status who received RT $(n=8)$ were not superior to those who did not $(n=2)$ (four-year EFS $87.5 \%$ vs $100.0 \%$ [95\% CI: $62.8 \%-100.0 \%$ ]; $P=0.62$ ) (Figure 2B), (fouryear OS $87.5 \%$ vs $100.0 \%$ [95\% CI: $62.8 \%-100.0 \%$ ]; $P=0.62$ ), albeit with a very small sample size.

\section{4| DISCUSSION}

Although limited by a small sample size, data from this study support the routine use of adjuvant RT in patients with FOXOl-positive disease even after complete resection. Prior studies in group I ERMS have failed to demonstrate a benefit to adjuvant RT. ${ }^{13}$ Given the biological similarity between FOXOl-negative ARMS and ERMS, findings from this study also suggest omitting adjuvant RT is rational for patients with group I, FOXOl-negative ARMS. Our findings add credence to risk stratification based on translocation status in the treatment of ARMS, as is being incorporated into contemporary studies. RT is not being offered to patients with group I, FOXOl-negative disease on the currently enrolling COG study, ARST1431, providing an opportunity to confirm this strategy in a prospective clinical trial.

Patients with ARMS and low-risk stage and group (stage 1 group I/II, orbital group III, or stage 2/3 group I/II) were eligible for enrollment on D9602, intended for low-risk RMS, prior to the opening of D9803 for intermediate-risk disease in $1999 .{ }^{17,25}$ Patients with lowrisk, fusion-negative ARMS treated on either D9602 or D9803 had 100\% EFS, suggesting the potential to reduce treatment intensity. ${ }^{25}$ Given the risk of secondary malignant neoplasms in patients with RMS treated with both an alkylating agent and RT, ${ }^{26}$ clinical trials have attempted to limit exposure to these therapies without affecting outcome, and controversy specifically remains on whether RT is necessary for patients with group 1 , FOXOl-negative disease. ${ }^{27}$ Although limited by sample size, the current analysis suggests eliminating adjuvant RT for patients with group 1, FOXOI negative may limit toxicities without affecting outcome.

Selected prior European cooperative group trials have attempted to limit RT by stratifying the use of RT by response to initial chemotherapy ${ }^{28}$ or after initial complete resection. ${ }^{29}$ The Cooperative Soft-Tissue Sarcoma Study Group trials 81, 86, 91, and 96 compared outcomes of patients with positive margins after initial complete resection treated with or without RT.

${ }^{29}$ RT was associated with improved local control, EFS, and OS especially for patients with ARMS; however, these studies did not account for FOXOl-fusion status and included nonRMS soft-tissue sarcomas. ${ }^{29}$ Because of differences in clinical staging or histologic classification between COG and European cooperative group data, we cannot compare our findings regarding the use of RT for patients with group I ARMS.

Prior IRS-COG studies suggested that RT dose reduction may be possible in patients with group I ARMS. Patients with group I/II ARMS were initially treated with 41.4 Gy on IRS III/IV. ${ }^{6,15}$ On D9803, treatment with 36 Gy yielded a local control rate of $90 \% .{ }^{14}$ In 
comparison, an analysis of IRS III/IV showed no statistically significant difference in local control and EFS in patients with group I stage I/II ARMS treated or untreated with RT. ${ }^{15}$ However, all prior analyses were limited by the lack of $\mathrm{FOXOl-fusion} \mathrm{data.}{ }^{30}$

We attempted in our current analysis to use FOXOl-fusion status to distinguish who would benefit from RT. The greatest limitation of our study is the small sample size; however, using available data of patients with group I ARMS treated on D9602, D9803, and ARST0531, our findings strongly support that RT improves outcome in patients who are FOXOI positive. Moreover, our findings support the concept of omitting adjuvant RT for patients with FOXOl-negative ARMS, as is the standard for patients with group I ERMS given that these patients have similar outcomes. ${ }^{9,10,13}$ Sequential studies have implemented RT at different times throughout therapy; however, differences in the timing of $\mathrm{RT}^{14,31-33}$ or dose escalation with hyperfractionation have not impacted outcomes. ${ }^{34,35}$ Phase $\mathrm{H}$ studies involving the use of proton therapy for patients with localized ERMS treated per D9803 or ARST0531 demonstrated promising similar five-year local control, EFS, and OS compared with those who received traditional photon RT with limited short-term side effects. ${ }^{36}$ Further data with longer follow-up are currently being acquired to determine if there are long-term benefits of protons over photons, which may also influence the overall discussion of RT in lower-risk patients.

The findings of our study are especially relevant for young children, with age being the most important factor influencing noncompliance with RT. ${ }^{37}$ For example, on D9803 and ARST0531, $12 \%$ and $8 \%$ of patients, respectively, did not receive RT per protocol. ${ }^{32}$ For patients with group II RMS treated on IRS I-IV, over half of operative bed recurrences were associated with noncompliance, especially omission of RT. ${ }^{38}$ In the current study, approximately $50 \%$ of patients $\leq 2$ years of age did not receive RT.

Overall, although our sample size precludes strong conclusions regarding RT for patients with group I ARMS, our study suggests the need for RT in patients with group I FOXOIpositive ARMS. Additionally, for patients with FOXOl-negative ARMS, our data in the context of its biological similarity to ERMS support the omission of adjuvant RT in these patients. The current COG intermediate-risk study, ARST1431, uses FOXOl-fusion status rather than histology to direct the use of adjuvant RT for patients with group I ARMS and will confirm this strategy with a prospective cohort (NCT02567435).

\section{Supplementary Material}

Refer to Web version on PubMed Central for supplementary material.

\section{ACKNOWLEDGMENTS}

Funding for this study was provided by Children's Oncology Group Grants U10CA180886, U10CA180899, U10CA098543, and U10CA098413; St. Baldrick's Foundation; and Seattle Children's Foundation, from Kat's Crew Guild through the Sarcoma Research Fund.

Funding information

Children's Oncology Group, Grant/Award Numbers: U10CA180886, U10CA180899, U10CA098543, U10CA098413; Seattle Children's Foundation; St. Baldrick's Foundation 


\section{Abbreviations}

$\begin{array}{ll}\text { ARMS } & \text { alveolar rhabdomyosarcoma } \\ \text { Cl } & \text { confidence interval } \\ \text { COG } & \text { Children's Oncology Group } \\ \text { EFS } & \text { event-free survival } \\ \text { ERMS } & \text { embryonal rhabdomyosarcoma } \\ \text { IRS } & \text { Intergroup Rhabdomyosarcoma Study } \\ \text { LF } & \text { local failure } \\ \text { OS } & \text { overall survival } \\ \text { RMS } & \text { rhabdomyosarcoma } \\ \text { RT } & \text { radiotherapy } \\ \text { VAC } & \text { vincristine, dactinomycin, cyclophosphamide }\end{array}$

\section{REFERENCES}

1. Perez EA, Kassira N, Cheung MC, et al. Rhabdomyosarcoma in children: a SEER population based study. J Surg Res. 2011;170(2):e243-e251. [PubMed: 21529833]

2. Skapek SX, Ferrari A, Gupta AA, et al. Rhabdomyosarcoma. Nat Rev Dis Primers. 2019;5(1):1. [PubMed: 30617281]

3. Raney RB, Anderson JR, Barr FG, et al. Rhabdomyosarcoma and undifferentiated sarcoma in the first two decades of life: a selective review of intergroup rhabdomyosarcoma study group experience and rationale for Intergroup Rhabdomyosarcoma Study V. J Pediatr Hematol Oncol. 2001;23(4):215-220. [PubMed: 11846299]

4. Maurer HM, Beltangady M, Gehan EA, et al. The Intergroup Rhabdomyosarcoma Study I. A final report. Cancer. 1988;61(2):209-220. [PubMed: 3275486]

5. Maurer HM, Gehan EA, Beltangady M, et al. The Intergroup Rhabdomyosarcoma Study II. Cancer. 1993;71(5):1904-1922. [PubMed: 8448756]

6. Crist W, Gehan EA, Ragab AH, et al. The Third Intergroup Rhabdomyosarcoma Study. J Clin Oncol. 1995;13(3):610-630. [PubMed: 7884423]

7. Lawrence W Jr., Anderson JR, Gehan EA, et al. Pretreatment TNM staging of childhood rhabdomyosarcoma: a report of the Intergroup Rhabdomyosarcoma Study Group. Children's Cancer Study Group. Pediatric Oncology Group. Cancer. 1997;80(6):1165-1170. [PubMed: 9305719]

8. Newton WA Jr., Soule EH, Hamoudi AB, et al. Histopathology of childhood sarcomas, Intergroup Rhabdomyosarcoma Studies I and II: clinicopathologic correlation. J Clin Oncol. 1988;6(1):67-75. [PubMed: 3275751]

9. Skapek SX, Anderson J, Barr FG, et al. PAX-FOXOl fusion status drives unfavorable outcome for children with rhabdomyosarcoma: a children's oncology group report. Pediatr Blood Cancer. 2013;60(9):1411-1147. [PubMed: 23526739]

10. Williamson D, Missiaglia E, de Reynies A, et al. Fusion genenegative alveolar rhabdomyosarcoma is clinically and molecularly indistinguishable from embryonal rhabdomyosarcoma. J Clin Oncol. 2010;28(13):2151-2158. [PubMed: 20351326]

11. Oeffinger KC, Mertens AC, Sklar CA, et al. Chronic health conditions in adult survivors of childhood cancer. N Engl J Med. 2006;355(15):15721582. [PubMed: 17035650] 
12. Breneman J, Meza J, Donaldson SS, et al. Local control with reduceddose radiotherapy for lowrisk rhabdomyosarcoma: a report from the Children's Oncology Group D9602 study. Int J Radiat Oncol Biol Phys. 2012;83(2):720-726. [PubMed: 22104356]

13. Wolden SL, Anderson JR, Crist WM, et al. Indications for radiotherapy and chemotherapy after complete resection in rhabdomyosarcoma: a report from the Intergroup Rhabdomyosarcoma Studies I to III. J Clin Oncol. 1999;17(11):3468-3475. [PubMed: 10550144]

14. Wolden SL, Lyden ER, Arndt CA, et al. Local control for intermediate-risk rhabdomyosarcoma: results from D9803 according to histology, group, site, and size: a report from the Children's Oncology Group. Int J Radiat Oncol Biol Phys. 2015;93(5):1071-1076. [PubMed: 26581144]

15. Raney RB, Anderson JR, Brown KL, et al. Treatment results for patients with localized, completely resected (group I) alveolar rhabdomyosarcoma on Intergroup Rhabdomyosarcoma Study Group (IRSG) protocols III and IV, 1984-1997: a report from the Children's Oncology Group. Pediatr Blood Cancer. 2010;55 \{4):612-616. [PubMed: 20806360]

16. Raney RB, Walterhouse DO, Meza JL, et al. Results of the Intergroup Rhabdomyosarcoma Study Group D9602 protocol, using vincristine and dactinomycin with or without cyclophosphamide and radiation therapy, for newly diagnosed patients with low-risk embryonal rhabdomyosarcoma: a report from the soft tissue sarcoma committee of the Children's Oncology Group. J Clin Oncol. 2011;29(10):1312-1318. [PubMed: 21357783]

17. Arndt CA, Stoner JA, Hawkins DS, et al. Vincristine, actinomycin, and cyclophosphamide compared with vincristine, actinomycin, and cyclophosphamide alternating with vincristine, topotecan, and cyclophosphamide for intermediate-risk rhabdomyosarcoma: children's oncology group study D9803. J Clin Oncol. 2009;27(31):51825188. [PubMed: 19770373]

18. Hawkins DS, Chi YY, Anderson JR, et al. Addition of vincristine and irinotecan to vincristine, dactinomycin, and cyclophosphamide does not improve outcome for intermediate-risk rhabdomyosarcoma: a report from the Children's Oncology Group. J Clin Oncol. 2018;36(2):2770-2777. [PubMed: 30091945]

19. M Dantonello T, Int-Veen C, Winkler P, et al. Initial patient characteristics can predict pattern and risk of relapse in localized rhabdomyosarcoms. J Clin Oncol. 2008;26(3):406-413. [PubMed: 18202417]

20. Rudzinski ER, Teot LA, Anderson JR, et al. Dense pattern of embryonal rhabdomyosarcoma, a lesion easily confused with alveolar rhabdomyosarcoma: a report from the Soft Tissue Sarcoma Committee of the Children's Oncology Group. Am J Clin Pathol. 2013;140\{1): 82-90. [PubMed: 23765537]

21. Fine JP, Gray RJ. A proportional hazards model for the subdistribution of a competing risk. J Am Stat Assoc. 1999;94:496-509.

22. Kaplan EL, Meier P. Nonparametric estimation from incomplete observations. J Am Stat Assoc. 1958;53:457-481.

23. Peto R, Pike MC, Armitage $P$, et al. Design and analysis of randomized clinical trials requiring prolonged observation of each patient. II. Analysis and examples. Br J Cancer. 1977;35(1):1-39.

24. Fisher RA. Statistical methods for research workers. 5th ed Edinburgh: Oliver and Boyd; 1934.

25. Arnold MA, Anderson JR, Gastier-Foster JM, et al. Histology, fusion status, and outcome in alveolar rhabdomyosarcoma with low-risk clinical features: a report from the Children's Oncology Group. Pediatr Blood Cancer. 2016;63(4):634-639. [PubMed: 26756883]

26. Heyn R, Haeberlen V, Newton WA, et al. Second malignant neoplasms in children treated for rhabdomyosarcoma. Intergroup Rhabdomyosarcoma Study Committee. J Clin Oncol. 1993;11\{2):262-270.

27. Borinstein SC, Steppan D, Hayashi M, et al. Consensus and controversies sies regarding the treatment of rhabdomyosarcoma. Pediatr Blood Cancer. 2018;65(2). 10.1002/pbc.26809.

28. Stevens MC, Rey A, Bouvet N, et al. Treatment of nonmetastatic rhabdomyosarcoma in childhood and adolescence: third study of the International Society of Paediatric Oncology-SIOP Malignant Mesenchymal Tumor 89. J Clin Oncol. 2005;23(12):2618-2628. [PubMed: 15728225]

29. Schuck A, Mattke AC, Schmidt B, et al. Group II rhabdomyosarcoma and rhabdomyosarcomalike tumors: is radiotherapy necessary. J Clin Oncol. 2004;22(1):143-149. [PubMed: 14701776]

30. Stevens M. Judgement required. Pediatr Blood Cancer. 2010;55:597598. [PubMed: 20589627] 
31. Michalski JM, Meza J, Breneman JC, et al. Influence of radiation therapy parameters on outcome in children treated with radiation therapy for localized parameningeal rhabdomyosarcoma in Intergroup Rhabdomyosarcoma Study Group trials II through IV. Int J Radiat Oncol Biol Phys. 2004;59(4):1027-1038. [PubMed: 15234036]

32. Casey DL, Chi YY, Donaldson SS, et al. Increased local fail ure for patients with intermediate-risk rhabdomyosarcoma on ARST0531: a report from the Children's Oncology Group. Cancer. 2019;125(18):3242-3248. [PubMed: 31174239]

33. Spalding AC, Hawkins DS, Donaldson SS, et al. The effect of radiation timing on patients with high-risk features of parameningeal rhabdomyosarcoma: an analysis of IRS-IV and D9803. Int J Radiat Oncol Biol Phys. 2013;87(3):512-516. [PubMed: 24074925]

34. Donaldson SS, Meza J, Breneman JC, et al. Results from the IRS-IV randomized trial of hyperfractionated radiotherapy in children with rhabdomyosarcoma- a report from the IRSG. Int J Radiat Oncol Biol Phys. 2001;51(3):718-728. [PubMed: 11597814]

35. Lin C, Donaldson SS, Meza JL, et al. Effect of radiotherapy techniques (IMRT vs3D-CRT) on outcome in patients with intermediate-risk rhabdomyosarcoma enrolled in COG D9803-a report from the Children's Oncology Group. Int J Radiat Oncol Biol Phys. 2012;82(5):1764-1770. [PubMed: 21470795]

36. Ladra MM, Szymonifka JD, Mahajan A, et al. Preliminary results of a phase 11 trial of proton radiotherapy for pediatric rhabdomyosarcoma. J Clin Oncol. 2014;32(33):3762-3770. [PubMed: 25332253]

37. Puri DR, Wexler LH, Meyers PA, et al.The challenging role of radiation therapy for very young children with rhabdomyosarcoma. Int J Radiat Oncol Biol Phys. 2006:65(4):1177-1184. [PubMed: 16682130]

38. Million L, Anderson J, Breneman J, et al. Influence of noncompliance with radiation therapy protocol guidelines and operative bed recurrences for children with rhabdomyosarcoma and microscopic residual disease: a report from the Children's Oncology Group. Int J Radiat Oncol Bio Phys. 2011;80(2):333-338. [PubMed: 20646841] 
A
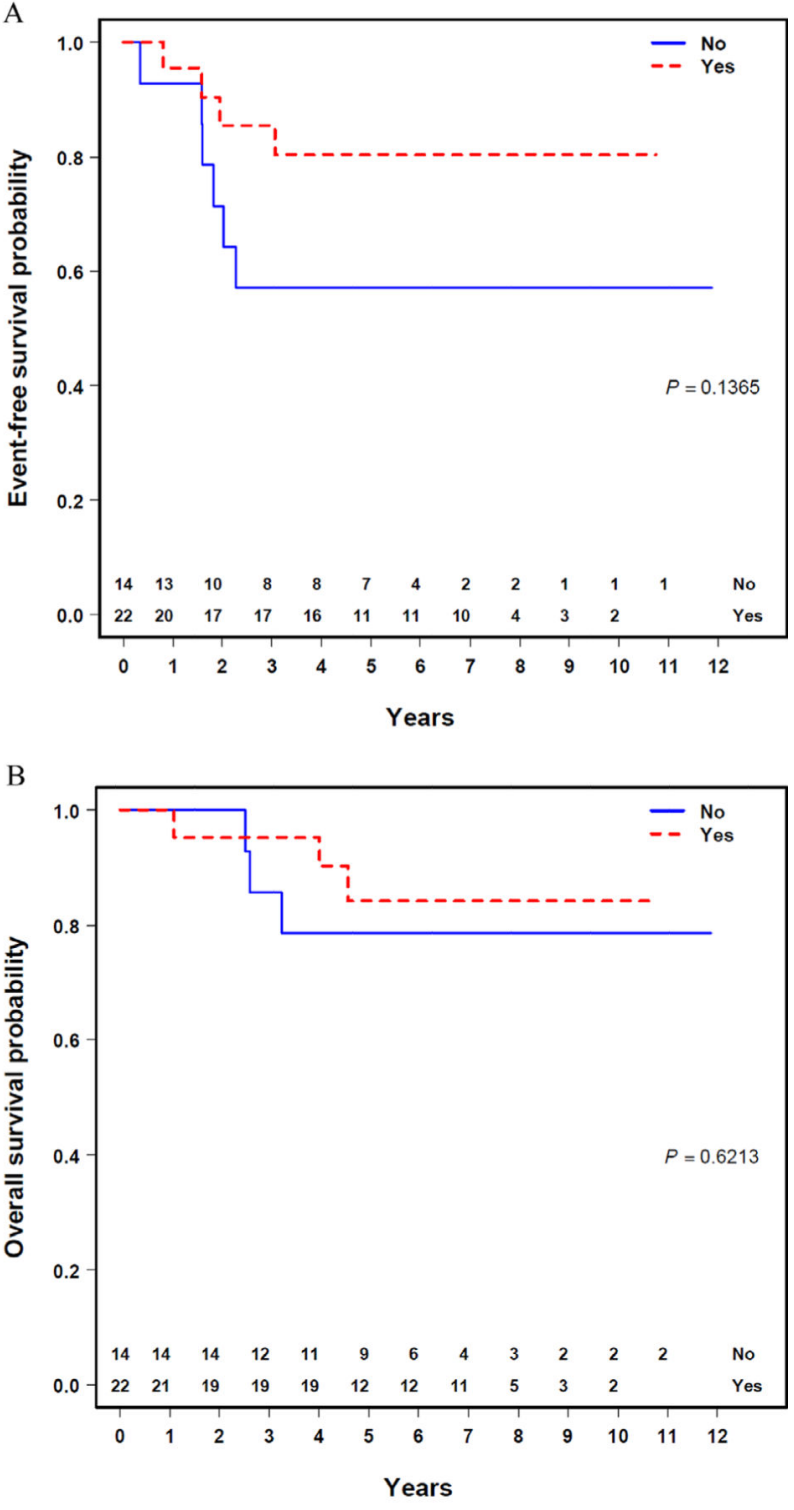

FIGURE 1.

Outcomes by use of radiotherapy 

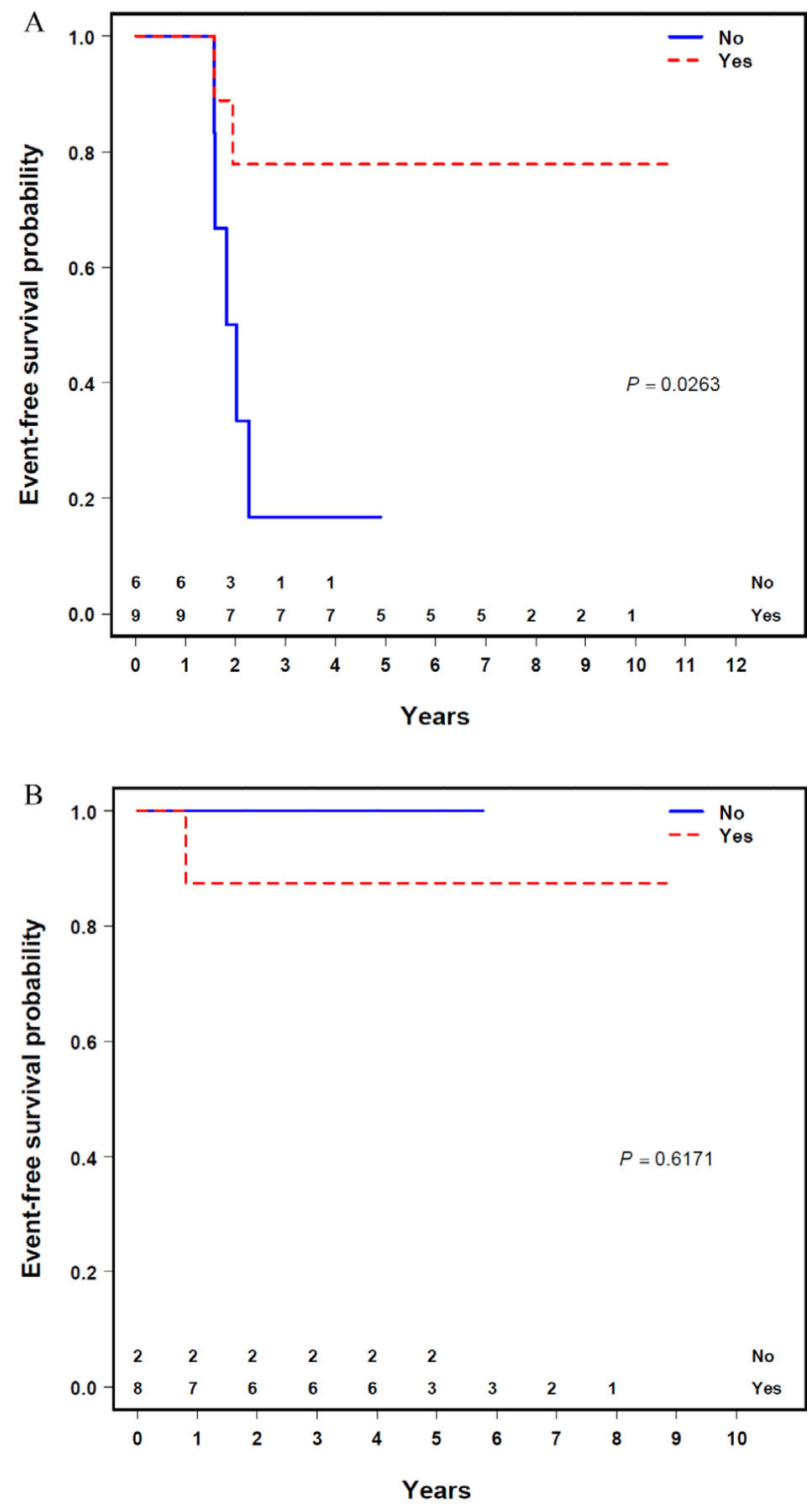

FIGURE 2.

Event-free survival by $\mathrm{FOXOI-fusion} \mathrm{status} \mathrm{and} \mathrm{use} \mathrm{of} \mathrm{radiotherapy}$ 


\section{TABLE 1}

\section{Clinical characteristics}

\begin{tabular}{|c|c|}
\hline Characteristic & $N(\%)$ \\
\hline \multicolumn{2}{|l|}{ Sex } \\
\hline Male & $24(67)$ \\
\hline Female & $12(33)$ \\
\hline \multicolumn{2}{|l|}{ Age, years } \\
\hline $\mathcal{2}$ & $9(25)$ \\
\hline$>2-10$ & $20(56)$ \\
\hline$>10$ & $7(19)$ \\
\hline \multicolumn{2}{|l|}{ Study } \\
\hline D9602 & $6(17)$ \\
\hline D9803 & $17(47)$ \\
\hline ARST0531 & $13(36)$ \\
\hline \multicolumn{2}{|l|}{ Radiation therapy } \\
\hline Yes & $22(61)$ \\
\hline No & $14(39)$ \\
\hline \multicolumn{2}{|l|}{ Stage } \\
\hline 1 (favorable site, $\mathrm{M}_{0}$ ) & $16(44)$ \\
\hline 2 (other site, $\leq 5 \mathrm{~cm}, \mathrm{~N}_{0}, \mathrm{M}_{0}$ ) & $14(39)$ \\
\hline 3 (other site, $>5 \mathrm{~cm}$ and/or $\mathrm{N}_{1}, \mathrm{M}_{0}$ ) & $6(17)$ \\
\hline \multicolumn{2}{|l|}{ Primary site } \\
\hline Head/neck & $7(19)$ \\
\hline Genitourinary, non-bladder/prostate & $9(25)$ \\
\hline Extremity & $11(31)$ \\
\hline Trunk & $8(22)$ \\
\hline Other & $1(3)$ \\
\hline \multicolumn{2}{|l|}{ Size } \\
\hline $5 \mathrm{~cm}$ & $26(72)$ \\
\hline$>5 \mathrm{~cm}$ & $10(28)$ \\
\hline \multicolumn{2}{|l|}{ FOXO1 fusion status } \\
\hline $\mathrm{FOXOI}^{-}$ & $10(28)$ \\
\hline $\mathrm{FOXO1}^{+}$ & $15(42)$ \\
\hline Unknown & $11(30)$ \\
\hline
\end{tabular}

Abbreviations: $\mathrm{M}_{0}$, no distant metastases; $\mathrm{N}_{0}$, regional nodes not clinically involved; $\mathrm{N}_{1}$, regional nodes clinically involved by imaging. 
TABLE 2

Clinical characteristics by use of radiotherapy

\begin{tabular}{|c|c|c|c|}
\hline & \multicolumn{2}{|l|}{$\underline{\text { Radiotherapy }}$} & \multirow[b]{2}{*}{$P$} \\
\hline & Yes $(N=22)$ & No $(N=14)$ & \\
\hline \multicolumn{4}{|l|}{ Age, years } \\
\hline$\underline{2}$ & 5 & 4 & \\
\hline$>2-10$ & 13 & 7 & 0.90 \\
\hline$>10$ & 4 & 3 & \\
\hline \multicolumn{4}{|l|}{ Study } \\
\hline D9602 & 1 & 5 & \\
\hline D9803 & 12 & 5 & 0.06 \\
\hline ARST0531 & 9 & 4 & \\
\hline \multicolumn{4}{|l|}{ Stage } \\
\hline 1 (favorable site, $\mathrm{M}_{0}$ ) & 5 & 11 & \\
\hline 2 (other site, $\leq 5 \mathrm{~cm}, \mathrm{~N}_{0}, \mathrm{M}_{0}$ ) & 11 & 3 & 0.003 \\
\hline 3 (other site, $>5 \mathrm{~cm}$ and/or $\mathrm{N}_{1}, \mathrm{M}_{0}$ ) & 6 & 0 & \\
\hline \multicolumn{4}{|l|}{ Size } \\
\hline $5 \mathrm{~cm}$ & 16 & 10 & 1.0 \\
\hline$>5 \mathrm{~cm}$ & 6 & 4 & \\
\hline \multicolumn{4}{|l|}{ FOXO1-fusion status } \\
\hline $\mathrm{FOXO1}^{-}$ & 8 & 2 & \\
\hline $\mathrm{FOXOl}^{+}$ & 9 & 6 & $0.40^{a}$ \\
\hline Unknown & 5 & 6 & \\
\hline
\end{tabular}

${ }^{a}$ Patients with an unknown fusion status were excluded. 